\title{
The Future Workplace: Views from the floor
}

Ann Davis*, Eddie Blass ${ }^{\dagger}$

*Work and Organisational Psychology Group, Aston Business School, Aston University, Aston Triangle, Birmingham, UK. B4 7ET

Corresponding author. E-mail address: a.j.davis@ aston.ac.uk

Tel.: +44 121204 3261; fax: +44 1212043327

${ }^{\dagger}$ Ashridge ${ }^{1}$, Berkhamsted, Hertfordshire, UK. HP4 1NS

\begin{abstract}
Speculation on the future of work and the nature of the future workplace has come to dominate much academic discourse in recent years. Rarely however has the voice of what might be termed the average skilled employee been heard; those who are still shaping a career and may be most at the mercy of whatever changes occur. This study seeks to fill this gap. Stemming from a one year research project at Cranfield School of Management, this paper focuses on data collected from a survey exploring the understanding of current and future organisation, and the nature of current and future leadership. The survey was carried out in 2003 and sampled 469 MBA graduands and a further 340 respondents to a web-based questionnaire. The paper provides an overview of the academic discourse on the future workplace, explores the perceptions and expectations of the sample and draws conclusions regarding significant anticipated trends for the future workplace as seen by those on the shop floor. These centre around increased flexibility and autonomy, but with limited awareness of the nature of leadership skills required to lead such a workforce.
\end{abstract}

\section{Keywords}

Leadership, organisational change, future workplace, line manager.

\footnotetext{
${ }^{1}$ Present address
} 


\section{Introduction}

Predictions regarding the future workplace predominantly stem from either concerns from relevant professional bodies (e.g [1-2]), from expert futurists (e.g. [3-4]), or from 'expert panel' studies (e.g. [5-7]). Throughout these works the themes of uncertainty and change continually appear. Rapid environmental, technological and social change has dominated the thoughts of management theorists for many years, with writers such as Rosabeth Moss Kanter, Tom Peters and Charles Handy arguing moreover that the pace of change continues to increase. Popular student texts on managing change have flourished in parallel, the more popular now being into a third and fourth edition since the early 1990s (e.g. [8-10]), and a cursory examination of both undergraduate and postgraduate business syllabi will almost invariably throw up a course on change. However, the focus of this approach tends to be on how to deal with "planned" or "emergent" change, not on the likely content of change or how organisations, and in particular their leadership, will need to adapt to possible futures.

There is of course a parallel literature on the nature of change in the business environment, a literature more commonly associated with strategy. A glance at the context of modern organisation with an eye trained in the business environment of the 1970s will instantly identify differences. The globalisation of business, the rapid increase in communications and other technologies, coupled with a shift in the west to a post industrial economy (where employment levels in the service economy vastly exceed those in industrial production) would render the workplace of the $21^{\text {st }}$ century recognisable, but recognisably different from that of 30 years ago [11-13]. That the business environment is different is undeniable. The nature of that difference is perhaps less tangible and tends to be caught up in the rhetoric of dominant political or social ideologies.

One theme that runs through much discourse about modern organisation is flexibility, necessary to deal more effectively with the uncertainty that characterises the work environment. This flexibility comes in a variety of forms and at a number of levels. At the organisational level, flexibility can manifest as a focus on niche rather than mass markets, creatively identifying and exploiting new opportunities and technologies, and adopting different forms of organising; from a post-Fordist melange of multifunctional teams, entrepreneurial, self regulating networks of production, to a cost-driven outsourcing of noncore activities, optimising use of organisational resources to accommodate those demands. Patterns of flexible employment may lead to increases in self-employment, subcontracting and other displacement strategies described by Atkinson more than 20 years ago as characteristic of the "flexible firm" [14].

The different forms of organising work may require individuals to become more (or less) involved in decision making, providing more (or less) discretion and accruing more (or less) responsibility for the quality of the output. The individual is also required to demonstrate personal flexibility either in terms of their skills or competencies, or their patterns of employment, or both. Felstead and Jewson [15] suggest an agenda of issues for flexible labour and non-standard employment, such that future workers will not be full-time, permanent, on open-ended contracts and secure. This may give rise to different labour markets; one characterised by skill recognition and development amongst professional and skilled technical employees, and others more open and externalised, where jobs are narrowly defined but tightly controlled unskilled and insecure and attract minimal training [16].

Functional flexibility, representing the development of new skills, abilities or competencies, implies enhanced learning, training and education, but increasingly becoming the 
responsibility of the individual employee. Gararan et al [17] for example see the individual focussing on the wider dimensions of employability implying an expectation of transience. Accepting that career is no longer hierarchical in a single organisation, but rather concerned with milestones related to enhanced competency the individual's focus shifts to selfemployment and/or careers spanning many organisations and a variety of job types.

Temporal flexibility also comes more to the fore in the globalised, virtual flexible work environment. Both the duration of employment and hours of work become negotiable, the power in the negotiation being in large part determined by the nature of the labour market. Cartwright [18] identifies that one eighth of people are either already self-employed or working on short-term or part-time contracts.

Tietze \& Musson [19] in a study of home workers across different industry sectors also highlighted a range of issues around work temporalities. The 9-5 day is rarely adhered to, and even when routines are intended, they are often interrupted by other household or social activities. The working day may continue late into the evening with home workers exercising both self-discipline and genuine autonomy and self-determination, often giving rise to highly effective use of available time. Their conclusion highlights the paradox of the home-work interface: while working at home unites work and home providing an opportunity for more integration, it simultaneously fragments work and home by introducing different temporalities into the private realm, making it harder to commit to either arena in a meaningful way.

These various changes in the nature of work and employment give rise to significant questions about the nature of management and leadership required in the flexible organisation. Managing remote workers will be quite different from the traditional roles of management and leadership. Similarly, managing flexible labour more broadly, including teleworking, franchising, zero-hours contracting and so forth will require a different approach from that adopted in traditional employment.

However we must beware being drawn unquestioningly into this managerial discourse. Flexibility is widely used as a rhetorical term, intended to imply something positive: flexible is good, rigid is bad. However, those who have the power to frame discourse can structure the perceptions and mindsets of those who wield power in organisations, while the reality for those on the receiving end may represent an alternative perception and mindset altogether. As far back as 1988 Anna Pollert [20] severely criticised the "flexible firm" accusing it, amongst other things, of being tied to a particular ideological agenda. However organisations and the bureaucratic form have a tendency towards rigidity which has on the whole served them well over many years. Moreover, stability, predictability, and security are basic to human psychology. Thus while the rhetoric of flexibility is so positive, the reality may be somewhat at variance.

Recently Nolan \& Wood [21] have reinforced a cautious approach to the "radical future" view, claiming that evidence to date does not suggest that careers are at an end or that tenure is falling, echoing Pollerts suggestions of the late 1980s. This however may be a reflection of the nature of their study, as despite identifying itself as study of the future of work, in reality it seeks to understand the present and recent past.

So far we have sought to outline some of the context for discussion of the future of work. In particular, we have emphasised the change and flexibility rhetoric as a core agenda. While 
being a dominant theme within management theory and education for some 20 years, the domain remains relatively poorly defined in terms of the direct implications for both leadership and organisation at a practical level. While predictions for wholesale radical change have been common for 20 years, experience indicates that these need to be treated with some caution. With that in mind, the next section goes on to examine in more detail some current predictions for the future of work.

\section{The future of work}

Richard Scase [22] in his portrayal of Britain in 2010 predicts some of the key characteristics being shaped by increased choice in how we live and work. In addition to the drivers identified above, he highlights mobility, independence, anxiety and risk, creativity, biotechnologies, socio-economic inequalities and the more fluid concept of identity as providing the opportunity for reinvention and development. Within the increasingly aging, singleton population, inequality is also set to continue. More people will be working in Indian restaurants than shipbuilding, steel manufacturing and coal mining combined, with the industrial and occupational structure changing such that massive supermarkets, the need for care assistants and other service oriented workers lead to low skill low pay jobs. At the same time he predicts a rise of non-standard employment in an entrepreneurial economy, moving towards the virtual corporation operating a psychological contract based on temporary loyalties.

Kulzick [23] claims that in the future, a successful business must focus on delivering real value to customers, but be able to anticipate and take advantage of rapid change in an increasingly complex, ethical and technical environment. Performance will be measured as a level of contribution to organisational goals, and organisational units will have to prove they are more efficient than external providers or else they may find themselves outsourced. Leadership in this different context will also need to adapt. Leaders will need to be responsive and adaptive and be able to build teams rapidly that they can trust and rely on. This is no easy task where transience is a defining characteristic. Equally teams will need to feel inspired and motivated to perform, as it will not be difficult for a team to make a leader look bad. If loyalty is only temporary, then leadership may be a temporary responsibility also. Harris [24] sees high-trust relationships as the key to this new organisational paradigm for virtual organisations which are dispersed both in place and in time, which is reiterated by Mirchandani [25] who cautions that work deliverables need to be forthcoming for the trust to exist and for teleworking to be successful.

Clampitt \& De Koch [26] identify the need to both surface and embrace uncertainty, a fundamental feature of leadership in the future. Currently, uncertainty is suppressed because people expect leaders to always be right and predict the future with precision in their desire to control events. Such prediction and control is unlikely to be possible in the future and unless expectations on leaders change, the conflict between the desire for certainty and the reality of uncertainty will result in leaders who are unable to manage in an uncertain world.

While this may not be what the sceptics are forecasting, the denial of the impact of increasingly connected global markets, over-capacity in most industries leading to lower profits, and confused and diminishing financial markets leads Minkin [27] to predict trouble ahead with our economic forecasting. For every one job lost in manufacturing or high tech, he claims an equivalent of 6.2 jobs are lost in the service sector. As such he claims that economic forecasting no longer works because the current wave of changes in the world are pushing us out of our traditional economic cycles. Care therefore needs to be taken in using 
predictions and forecasts that are based on extrapolating trends based on economic cycles as the certainties of the past may not be certainties in the future.

In light of this apparently increasing unreliability of traditional forecasting approaches, it is valid to consider other sources of knowledge regarding the firm's future. In particular, the reliance of "top-down" approaches to strategy has been challenged recently by the "resourcebased view" and the rise of the competency movement generally (e.g. [28-30]). Senge [31] questions whether we cling to the view that only the top can initiate change in order to protect ourselves. He claims that hierarchical authority drives compliance not commitment and top management initiatives often back fire. Salisbury [32] extends this view, suggesting that 'members of the organisation need to replace the old mental model of getting paid for what they do with a new model where they believe they are paid for creating and sharing knowledge.' This makes embracing uncertainty everybody's responsibility rather than simply that of those in management and leadership roles. It also provides a significant justification for exploring the understanding and expectations of the average skilled employee in relation to their views on the future organisation.

The question arises as to whether bottom-up initiatives would be allowed to succeed by top management. Van Marrewijk \& Timmers [33] see two developments moving organisations from authoritarian and custodial models of management to collegial and motivational models. These are work-life balance and the business case, with dialogue from non-dominant positions being the key (see also [34]). Such a non-dominant stance would support the views of Seltzer and Bentley [35] who see creativity and risk management as the skills for the new economy rather than command and control. They identify risk as a combination of futures thinking, decision making, stress management and learning from failure. Creativity can be encouraged by the creation of a learning environment which establishes trust, freedom of action, variation of context, the right balance between skills and challenge, interactive exchange of knowledge and ideas, and real world outcomes.

In summary, words such as flexibility, globalisation, digitalisation and so forth are rife in management journals and magazines, but the extent to which such rhetoric is actually held as the reality for the future by managers in organisations has not been tested. This study set out to explore the current experience and future expectations of existing employees regarding the nature of work organisation and leadership. As noted earlier, the study's unique contribution is the broadening of a futures oriented methodology to non-futures and non-academic respondents, who nevertheless have a clear and direct interest in the future of work. These respondents are the managers who will both be responsible for creating, leading and managing change over the next 10 years and also will have to position themselves in the new reality that their vision of the future represents.

\section{Methodology}

These data stem from a research project at Cranfield School of Management looking at the future of management and leadership. In order to establish a vision of the future of management and leadership, a vision of the future of work and the workplace needed also to be established. To this end, the research reported here was carried out.

The core of the research was a web-based questionnaire, which focussed on 2 time frames; respondents initially were asked to report on their experience of the current workplace and the key skills managers and leaders need to operate effectively within it. They then reported on their expectations of the nature of the workplace in 10 years time and the managerial and 
leadership skills that would be needed to operate effectively there. Finally, in encouraging more direct comparison between the two timeframes, respondents were asked to identify those issues, skills and activities that would be less important in 10 years time.

Two groups of respondents were sought. The first were Cranfield MBA alumni, the survey being hosted on the alumni website for 6 weeks from April 2003. An e-mail introducing the survey was sent to MBA alumni identified by the Trident database (excluding current students and alumni who have requested not to receive circulars) at the end of March 2003. A total of 3,000 e-mails were sent, and 469 useable responses were received (after the data were screened for repeat submissions, etc) giving an approximate response rate of $15 \%$

The gender mix of the alumni respondents was $401(86 \%)$ males to $65(14 \%)$ females, this being broadly representative of the population ratio. The sample represented a diverse range of nationalities. Slightly more than three quarters of the sample were British (77\%), with a further $9 \%$ being non-British Europeans. Africa, North America, South America and the Far East were each represented by approximately $2 \%$ of the sample, with $1.5 \%$ coming from the Indian Sub-Continent. The remaining 4.5\% were from Australia and New Zealand.

In addition, a second group of respondents were canvassed. In line with the broader aims of the overall project, a general questionnaire was posted on the internet, available to all but advertised directly via a number of futures and professional listings. While its primary aim was to conduct a skills audit for the future, it also included the five core questions described above to orientate respondents appropriately for the questionnaire as a whole. An additional 340 usable responses were received during a 2 month period. $75 \%$ of these respondents were employed in the private sector, a further $11 \%$ worked in the public sector, and $9 \%$ were selfemployed. The remaining 5\% were either students, operating in the voluntary sector or classified as "other".

Initially, the responses of only the alumni were used to generate a framework for constructing an image of the current and future workplace and its leaders. The issues identified by respondents of characteristics of the relevant focus were transferred to cards and independently a futures specialist and an occupational psychologist sorted the responses into meaningful groups. The two professional allegiances allowed two different perspectives to be brought to the data. Subsequently, an agreed sort structure was negotiated between the experts. As the data from the web-based survey sample was received it was similarly sorted, however the initial structure emerging from the alumni sample appeared to represent a more coherent illustration, and therefore the more open sample data were re-sorted along the original alumni sample structure. The data presented below are the results of these analyses.

The results are presented in three sections. First, respondents' views of the current and future workplace are identified contrasted and discussed. Second, views about the nature and skill requirements of current and future leaders are similarly analysed. Finally, those aspects of the workplace and leader behaviour that were felt likely to decrease in importance are presented and discussed in light of the preceding discussions.

\section{Current and future workplace}

The items provided by respondents when asked to describe first the current workplace and subsequently the future workplace were ultimately sorted into five groupings each. The same five groups were identified for both the current and the future workplace and therefore facilitate direct comparison. These five groupings represented: 
- the nature of work, including complexity, technology, innovation and entrepreneurialism

- the nature of the work environment, particularly external influences and the nature of regulation

- the organisation of work, addressing the tensions of bureaucracy versus flexibility, and specialist versus generalist roles

- $\quad$ cultural elements, dealing primarily with issues of politics, pressure, stress and support, and

- $\quad$ individual impact on the workplace, including the role of flexible teams, control over work, and wider issues of ethics at work

While these groupings emerged for both time frames, there was a clear vision of change occurring over the ten year timeframe.

In terms of the nature of work, the current workplace was described by many as challenging, either through a drive for innovation and entrepreneurialism, or in contrast through a lack of creativity, characterised by specialist expert roles and functional knowledge. By contrast, the future workplace was seen to be more distributed. Facilitated through technology, a multiskilled, information heavy and project based environment was predicted to meet customer needs. Short-termism while present across both frames decreased in frequency in the future time frame from the current. Overall respondents tended to see a positive future.

With regard to the work environment, the complexity, diversity and fast paced dynamism currently experienced by people is expected to diminish, as the virtual, fluid and instant workplace evolves. Uncertainty, confusion, unpredictability and unethical all are words used to describe the current workplace, while interdependent, efficient, and increasingly global describe the workplace in 10 years time.

The idea of the 24:7 workplace which is outsourced to free-lancing portfolio workers is offered in the future workplace in terms of the organisation of work, while the current workplace is described by words such as bureaucratic, meritocratic, leaderless, traditional, hierarchical, matrix and results oriented. The future, on the other hand, is described as remote, dispersed, knowledge-led, hybrid and results rather than system focussed. The virtual workplace enabling self determination and liberation from bureaucracy so beloved of theorists for the past 30 years remains just over the rainbow.

In terms of the cultural elements, the top three issues for both time frames are the same, although in different orders. The current characterisation of a stressful culture in a fast paced and competitive setting, shifts to becoming first competitive then fast paced and subsequently stressful. This again suggests an optimistic outlook, the changes in technology and shifts away from bureaucracy towards greater autonomy perhaps enabling people to develop and express their creativity positively. The ethical landscape also appears to improve, with a decrease in concerns over office politics, macho working hours and support for cultural diversity. This is some distance from the typical current landscape described as political, aggressive, and unsupportive although it should be noted that to some respondents, the current environment was felt to be warm and inspiring. Interestingly, the explicit social dimension of work largely disappears from visions of the future.

The impact that the individual will have on the workplace is based on flexibility in both time frames, but more so in the future than present. Teams, greed, lack of loyalty and the 
impersonal nature of work impact on the individual currently, while they expect mobility, work-life balance, competence, teams, ageing and isolation to impact in the future.

\section{Current and future leaders}

When asked to describe 'leadership' both now and in 10 years time, again five clusters of issues emerged. These were:

- Interpersonal elements, dealing with how leaders handle interacting with others

- Knowledge and skills, those capabilities that leaders need to function effectively

- $\quad$ Fitness to lead or leadership capabilities, features associated with the visionary aspect of leadership, providing direction and motivation, forward looking and problem solving

- $\quad$ Personal outlook, the way in which a leader constructs his or her interactions with the environment, how they engage with the world around them, and

- $\quad$ Personal disposition, akin in part to personality attributes, but encompassing issues of values and beliefs.

The key similarities between the two timeframes were the centrality of interpersonal skills and communication skills as defining effective leadership. Indeed, there was much less variation in the responses from the current to future leader issues than between the current and future workplace, despite the clear differences identified in the nature of work organisation identified earlier. Overall, respondents seemed to find it more difficult to identify the types of leaders or leader behaviours that the future workplace would require. This to an extent mirrors the relative lack of detail in the literature pertaining to future leadership requirements as against future work environment. Whether the reason for the relative lack of detail and consensus on these issues stems from no easily accessible rhetoric, or a genuine lack of appreciation of what might be required is unclear. Still, important differences between the assessments at the different time points are identifiable as detailed below.

The interpersonal elements were perhaps the dominating features of the construction of leadership and leadership behaviour at both time points, with communication skills being seen as chief amongst these. In addition, team skills, developing or coaching others, and politics or diplomacy were all mentioned in both time frames, although with greater frequency in the current time frame than future. Networking or being connected and cross cultural skills were also mentioned in both time frames but were more frequently mentioned in terms of the future. These patterns seem to mirror the construction of work identified above, the current workplace being more concerned with politicking while the future workplace becomes more networked and interdependent. Interestingly, listening and negotiating were mentioned in the current time frame but missing from the future, perhaps further echoing the relative absence of social organisation identified in the future workplace. Outsourcing relationships were foreseen for the future but not seen as relevant now.

Key leader knowledge and skills were clearly focussed around people skills first in both time frames. Beyond that, and again echoing the nature of the workplace, for current leaders functional or expert knowledge was the second most often cited issue whereas for future leaders IT skills were much more of a priority, with functional/expert knowledge featuring much further down the list of priorities. Perhaps surprisingly, being multi-skilled while currently significant, dropped out of the vision of the future leader altogether. Whether this was oversight, or actively chosen to be unimportant, or because it becomes so essential as to be self evident cannot be determined. New areas to appear in the future are cross-functional, 
cross-industry, knowledge management and learning skills, these perhaps being more generic abilities than specific skills. Time management, diversity, planning, influencing and project management all remain much the same for both time frames, while the importance of cost control and financial skills appears to diminish in the future.

Fitness to lead or leadership capabilities did not significantly vary over time. Vision was key for both time frames (although it was mentioned more often in the current context than the future) followed by strategic thinking, leadership skills, motivating others and change implementation. Cited with lesser frequency but again consistent across time frames were decision making, prioritising, foresight, delegating, empowering, and inspiring. Fast thinking, problem solving, adaptability and credibility all appeared in the current time frame only, while commitment, and virtual team management appeared only in the future.

Words describing a leader's personal outlook were more popular generally in the current time frame than future. This is the grouping with the greatest amount of difference with only work-life balance, focus/clarity and open mindedness appearing in both time frames. The current time frame included the descriptors creative/new ideas, analytical, entrepreneurial/initiative, and consistent/fair. The future suggested customer oriented and ethical as the features of a leader's personal outlook.

Again, differences were found in the words used to describe personal disposition. Flexible was top for both time frames, but the current time frame then emphasised sensitive/empathetic, integrity/honesty, self awareness, resilience and confidence to a greater degree than they appeared in the future time frame. Self-motivated, coping with information overload and international scope all appeared in the current time frame only, while trust building and coping with uncertainty only appeared in the future time frame.

\section{Less of in the future}

Those aspects that respondents expected to see less of in the future were clustered under 4 headings:

- $\quad$ knowledge and skills, the depth and degree of specialisation in particular

- $\quad$ cultural elements, while not necessarily being less prevalent, at least being less significant in a move towards a global business environment

- $\quad$ personal elements, reflected the shift towards more flexible employment patterns and the implications for loyalty and direct interaction

- $\quad$ organisational elements, reflecting the suggested move towards location free employment and associated changes to structures and authority

Fewer participants provided responses to this question, and those that did tended to provide fewer suggestions than they did for the earlier items.

In terms of the knowledge and skills which were highlighted as having less of in the future, in depth knowledge and expertise came out top, with specialist areas and "number crunching" subjects coming next. Functional skills, IT skills and languages were also mentioned.

Qualifications in general are expected to be less significant, the MBA in particular appearing to take something of a hit. Perhaps in contrast to earlier findings, interpersonal skills also were suggested as being less relevant. This however may relate to the organisational issues associated with more distant or location free work and the greater use of computer-mediated interaction. 
In terms of cultural elements, the nationality, background or race of colleagues was anticipated to become less important. Given the increase in internationalisation identified earlier, it is surmised that this should be interpreted as a move towards acceptance of diversity rather than increased homogeneity of the workforce. The "macho" culture of the workplace highlighted in response to the first question also is expected to decline; office politics, targets, profit focus, and "presenteeism" all being suggested to reduce in importance.

Organisationally, the sample expect to see less location/office buildings, hierarchy and command \& control bureaucracy, as well as less work, employment, supervision and paper. Coupled with this, a reduction in business travel and a reduced requirement for physical presence at the workplace were also mentioned. This "distancing" trend is further reflected in relation to the personal elements identified. The sample expect less loyalty to organisations, less face to face contact, and indeed fewer people actually having jobs. Of those remaining in employment, age, past experience and ability to conform all seem to be issues that are felt likely to reduce in importance over the coming decade.

\section{Themes emerging from the floor}

A number of themes seem to emerge from these data. First, and in line with the introduction, there is a clear expectation of a significant change in the future away from the "company worker" towards a more flexible, responsive, autonomous and widely skilled professional. These professionals will be operating in an environment which has been transformed in order to accommodate their perspective on life; control systems will be loosened, bureaucracy and politics will be greatly reduced and work will become fun.

The responses generally to the survey seem to indicate optimism about the future of work. The nature of the work "challenge" seems to be one that respondents view positively, or at least feel better able to cope with. The data suggest a shift from a structured, ordered workplace towards a more flexible, hi-tech, autonomous workplace. While at present the development of technology is felt to be challenging, by 10 years in the future technology will become simply a tool to facilitate more flexible forms of work organisation, with the upcoming generations ever more IT literate.

In contrast, the implied decrease in a short-term perspective is puzzling, as the increase in portfolio workers, outsourcing and project work predicted would, logically, lead to an increase in short-term work assignments. Maybe this is taken for granted by some and therefore not worthy of mention, or perhaps it reflects a belief that companies will have pared down to the core, so those left in the workplace will be fairly secure in their (flexible) jobs.

The globalisation and digitalisation of the work environment appears inevitable, although the pace of change in 10 years time is predicted not to be as fast as it is now. A lot of the unpredictability and volatility will have dissipated, and a more stable environment is predicted, or perhaps hoped for. The data suggest implicit support for a "punctuated equilibrium" understanding of change, rather than a continuous transformational view. It may be that the view is one of a step-change, beyond which we will emerge into a more stable but different way of working which is more acceptable and amenable to most.

Structure seems to be at the heart of what is "bad" about organisation at present and what will be "good" in the future. The words used to describe the organisation of work now are a range of different organisational structures, from hierarchical, bureaucratic and traditional, to matrix, specialist and cost oriented. In the future, the words offered paint a picture of work 
being organised in a more autonomous manner, with workers being remote and dispersed, there being more focus on people skills, less structure in smaller companies, and people working freelance, outsourced and 24:7. If people have more freedom about how they choose to organise their work allocation, then the boundaries of 9-5 will shift towards 24:7 naturally as choices over which hours to work and which to undertake other activities give people the flexibility to plan their days accordingly.

There is a natural flow of logic towards the future workplace being less stressful. Certainly many people find the journey to work at the start of the day one of the most stressful elements of the day. In the more flexible, home-based future, this major stressor can be removed, and hence people should cope better with the pressure of the work itself. The current workplace culture is described in two ways, either as an aggressive, insecure, competitive culture, or as an exciting, supportive and stimulating culture. Both are fast paced, hectic and stressed, but around half the sample offer positive descriptors while the other half negative. The future seems much more positive with autonomy, self-determination, and diversity becoming the watchwords, while all the negative descriptors disappear from the list except for aggressive which is only foreseen by a third of those who feel it is present now.

While people use words such as unhappy, greedy, selfish and impersonal to describe the mood of the current workplace, the most frequently cited issue, both now and more so in ten years time, was flexibility. A shift towards descriptors such as mobile, competence, ageing and work-life balance takes place, implying a significant change in values underpinning work and the nature of organisation. Not all views are positive, there is some concern about becoming isolated or lonely in the future, by virtue of the distant relationship with the organisation and colleagues, which is only a very minor concern now. Autonomy may suit many, but others prefer teams and a sense of belonging to an organisation. Who controls the flexibility will also impact greatly on individuals. If the organisation dictates how they want the individual to be flexible, then the benefits of flexibility to the individual disappear.

In relation to the nature of the leadership now and in the future, there appears to be a shift from models commonly associated with "management behaviour" towards those more commonly described as "leadership characteristics". With respect to their interpersonal dealings, while currently there is a team focus and developmental feel to the words used (e.g. listening, coaching), the transactional nature of negotiation and diplomacy and a focus on process as much as outcome seem as important in the future. In addition, interpersonal descriptors are focussed on their personal achievement and success, so being connected/having networks is seen as important in a team-working output-driven future environment.

This is also reflected in the knowledge and skills that are mentioned with regard to the current time frame. People management skills and functional or expert knowledge are central to this construct with the other general management skills making up the remainder of the construct (cost control/finance, organisational skills, time management, project management, planning, computer understanding and so forth.). The focus is on how the leader would go about leading or managing a task rather than being about the nature of the leader themselves. The future leader seems less concerned with process and more concerned with output. This translates into knowledge and skills based around learning, cross-functional/cross-industry understanding, and the ability to manage themselves and their success through influencing, analysis, time management and project co-ordination. 
In terms of their leadership capabilities, the current leader is expected to have the visionary, strategic thinking and leadership skills that motivate others and inspire them to follow, while at the same time having the more down to earth management skills of decision-making, anticipation, prioritising, problem-solving, fast-thinking and being adaptable to be able to get themselves out of a hole. They also need to be credible and able to implement change, the latter of which may depend on the former. Future leaders' capabilities are about finding a way forward and ensuring that they get there. As well as having the vision, inspiration and foresight of the current leader, they will also drive change, manage virtual teams, achieve outcomes and gain commitment from others. The future leader knows where they are going and will make sure that people follow them there. Achievement motivates them to lead. They are focussed on the outcome.

There seems to be an expectation that the current leader is somehow entrepreneurial and creative, perhaps leading into greener pastures, while also being the open minded, consistent rock on which people can depend and rely. The words that fall into the personal outlook category almost dare the current leader to do something different, but at the same time they are restricted to doing it in a more traditional, bureaucratic way, by being analytical, focussed and balanced. The personal outlook of the future leader is outcome-focussed and customer oriented. However, here we also see a shift in underlying values; the future leader will be ethical and open, and concerned with their work life balance. Whether they are or not, the future leader will truly believe that they are right.

Finally regarding the disposition of current and future leaders, at present sensitivity and empathy to others' needs are balanced by self-awareness, self-motivation, confidence and ability to cope with information overload. In terms of their potential, they need to have international scope and remain resilient to stress and pressure. In the future, resilience and the ability to cope with uncertainty are added to self-confidence and flexibility. Trust building, honesty and the integrity that underpin their self-belief, will drive them to make the decisions that they do.

The issues raised with respect to what respondents expect less of in the future support the earlier findings. Independent, autonomous portfolio workers being outsourced with little supervision, bureaucracy or face to face contact supports the idea of working at home, remotely and virtually. The predicted reduction in concern about age, nationality etc suggests that the 'diversity' message is getting through, and the shift away from profit and costs suggests that wider ethical considerations are starting to make an impact on business practice.

The proposed move towards greater generalist knowledge is perhaps surprising as portfolio workers traditionally specialise in one particular area of expertise and sell that expertise to more than one organisation. Generalists leading a portfolio lifestyle might reflect the current uncertainty in the labour market rather than a significant trend for the future. The current labour market is very volatile and uncertain, with people being expected to turn their hand to many different things in order to remain employable in organisations. Once the "step change" of technology is passed and the (punctuated) equilibrium is restored in the shape of a more flexible, remote model, this need for everyone to be able to do everything may settle back to allowing people to be experts in their fields.

Equally, the shift away from qualifications is interesting as there is a shift towards lifelong learning. The need to get learning 'badged' in the future may not be as important as it is 
now, while actual competence and proven ability are likely to be more popular measures of learning than certificates.

\section{Conclusions - trends for the future of work}

The current workplace does not appear to be a happy place. It is seen as extremely challenging, fast changing and stressful, where the organisation of work is dominated by the structure of the organisation, and the work environment is in a state of flux. The command and control picture painted by Nolan \& Wood (2003) as the vision for the future appears to the current reality for the sample in this study.

In 10 years time, this is predicted to have changed, but given the negativity of the current situation, it is questionable whether this is simply wishful thinking. The future workplace is seen as being hi-tech, virtual and global, diverse, competitive but autonomous, and people will be organising their own work patterns to fit their desired lifestyle. Generally the tone of words used to describe the future are more positive than those for the current time frame, but the reality of whether or not this is actually a more positive work experience will only be seen with time.

Given this substantial shift in the workplace, it is surprising that the sample's view of leadership needing to change is less marked. Interpersonal skills are still predicted to be the most important leadership skill, although virtual team management and managing outsourced relationships appear as new areas for consideration. Ethics are also predicted to be more important in the future, and empathy will give way to the ability to build trust. Trust was a theme that also came through in the literature review.

As there will be fewer physical manifestations of organisations, they will rely on autonomous individuals networking to create a sense of organisational identity and culture, rather than a prescribed structure and environment influencing how people are working. Quite how effective this will be or how it will work in practice, nobody knows, but historical examples such as Tupperware, Avon, etc suggest that much can be achieved in such dispersed structures.

Such dispersion in itself poses new challenges for the future leader which are perhaps underappreciated by the sample and their predictions for what the future leader will look like. There are few organisations that are truly operating in this way at the moment, so it is difficult to get a feel for what such leadership will involve. Some organisations have some areas working to this pattern, but they tend to be led by 'pioneers' in their fields who are as much motivated by operationalising a new way of working as they are by the work itself. How this will transfer to mainstream organisational practice is questionable, but certainly the sample sees this as happening, as they describe the current workplace in such unstable terms. While the literature on home-working shows what would currently be regarded as a lack of discipline, it also shows it can be an efficient way of achieving organisational goals.

Certainly there does not appear to be a uniform skilled labour market appearing in the future. On the one hand there seems to be the development of outsourced, portfolio workers who are paid for achieving outcomes, with temporary loyalty to whoever is paying the next invoice, and on the other there are more permanent, remote teams where trust and loyalty still seem to be concerns. These two are not mutually exclusive. It follows the current trend towards smaller, leaner organisations with a generalist core, who might then employ specialist expertise on a freelance basis as they need it. As the percentage of the population gaining 
degree level education continually increases, it is possible that a degree will become the minimum entry level to the core of the organisation. After some time in a couple of organisations, these graduates might prefer to specialise in an area and shift to the portfolio labour market for greater flexibility and financial gain.

Whatever the future might hold, the views proffered by a general management population sample both support and contradict the views of the experts. They support the view of how the workplace and nature of work is changing, but are less prolific on what this means in terms of management and leadership practice. Perhaps they are more focussed on the core skills underlying management and leadership practice than buying-in to a new model for the future. Perhaps they don't really see the need for that much to change in the way that they work, even though the context of the work will itself change. Perhaps there is an element of denial and wishful thinking, as they are clearly highly stressed and challenged at the moment, and wish to be less so in the future. Whatever the reason, the results give pause for thought as to whether or not the literature on the future of work is driving a different change agenda to the reality on the floor.

\section{Acknowledgement}

This work was carried out at the Cranfield School of Management whose support in this research is acknowledged. 


\section{REFERENCES}

[1] Hitt MA $21^{\text {st }}$ Century Organisations: Business Firms, Business Schools and the Academy. Academy of Management Review 1997;23(2):218-224.

[2] Tate WV Futures Project: Implications of futures studies for business, organisations, management and leadership. UK: CEML, 2000.

[3] Curtain R. The Workplace of the Future: Insights from futures scenarios and today's high performance workplaces. Australian Bulletin of Labour 1998; 24(4):279-294.

[4] Didsbury Jr HF (Ed.) $21^{\text {st }}$ Century Opportunities and Challenges: An age of destruction or an age of tranformation. Maryland: World Future Society, 2003.

[5] Business Schools Advisory Board The Contribution of the UK Business Schools to development Managers and Leaders. UK CEML; 2002.

[6] De Jager P, De Vries J. Global Organisations. London: The Global Future Forum, 2002

[7] Non-HE Advisory Board Management and Leadership: Building the Future Supply. UK: CEML, 2002.

[8] Burns B. Managing Change (4th Edn.) London: FT Prentice Hall, 2004.

[9] Carnall CA. Managing Change in Organisations, (4th Edn.) London: FT Prentice Hall, 2003.

[10] Senior B. Organisational Change (2nd Edn,) London: Prentice Hall, 2002.

[11] Kompast M, Wagner I. Telework: Managing spatial, temporal and cultural boundaries. In Jackson PJ, Van Der Wielden JM (Eds) Teleworking: International Perspectives. London: Routledge, 2004.

[12] Thompson P, Warhurst C. (Eds) Workplaces of the Future. Basingstoke: Macmillan Publishing, 1998.

[13] McDonald JW. The Global Impact of IT: The Connected Versus The Unconnected. In Didsbury Jr. JF. (Ed) Frontiers of the $21^{\text {st }}$ Century: Prelude to the new millennium. Maryland: World Future Society, 1999.

[14] Atkinson J. Manpower strategies for the flexible organisation. Personnel Management 1984;August:28-31.

[15] Felstead A, Jewson N. Flexible Labour and Non-Standard Employment: An agenda of issues. In Felstead A, Jewson N. (Eds) Global Trends in Flexible Labour. Basingstoke: Macmillan Publishing, 1999.

[16] Hendry C. Human Resource Management: A Strategic Approach to Employment. Oxford: Butterworth-Heinemann, 1995.

[17] Gararan TN, Morley M, Gunnigle P, Collins E. Human Capital Accumulation: the role of HRD. Journal of European Industrial Training 2001;25(2):48-68.

[18] Cartwright S. New forms of work organisation: Issues and Challenges. Leadership and Organisation Development Journal 2003;24(3):121-122.

[19] Tietze S, Musson G. When 'Work' Meets 'Home': Temporal flexibility as lived experience. Time and Society 2002;11(2/3): 415-334.

[20] Pollert A. (1988) The "flexible firm": fixation or fact? Work, Employment and Society 1988;2(3):281 - 316.

[21] Nolan P, Wood S. Mapping the Future of Work. British Journal of Industrial Relations 2003;41(2):165-174.

[22] Scase R. Britain in 2010: The new business landscape. Oxford: Capstone Publishing Ltd, 2000

[23] Kulzick RS. Managing in the Future: What management skills will be needed? In Didsbury Jr HF. (Ed) 21st Century Opportunities and Challenges: An age of destruction or an age of tranformation. Maryland, USA: World Future Society:2003. 
[24] Harris M. Rethinking the virtual organisation. In Jackson PJ, Van Der Wielden JM. (Eds) Teleworking: International Perspectives. London: Routledge, 1998.

[25] Mirchandani K. No longer a struggle? Teleworkers' reconstruction of the work non-work boundary. In Jackson PJ, Van Der Wielden JM. (Eds) Teleworking: International Perspectives. London: Routledge, 1998.

[26] Clampitt PG, De Koch RJ. Embracing Uncertainty: The Essence of Leadership. Armonk New York: M.E.Sharpe, 2001.

[27] Minkin, B. H. (2003) Keep the CON in Economics: Why economic forecasting doesn't work. In Didsbury Jr, HF. (Ed) 21st Century Opportunities and Challenges: An age of destruction or an age of tranformation. Maryland, USA: World Future Society, 2003

[28] Prahalad CK, Hamel G. The core competence of the corporation. Harvard Business Review 1990;68(3):79-91.

[29] Hamel G, Prahalad CK. (1994) Competing for the future. Harvard Business Review 1994;Jul/Aug:122-128

[30] Boxall P, Purcell J. (2003) Strategy and Human Resource Management, Basingstoke: Palgrave MacMillan, 2003.

[31] Senge PM. Leading Learning Organisations: The Bold, the Powerful and the Invisible. In Hesselbein F, Goldsmith M, Beckhard (Eds) The Leader of the Future. San Francisco: Jossey-Bass Publishers, 1996.

[32] Salisbury M. Creating a process for capturing and leveraging intellectual capital. Performance Improvement Quarterly 2000;13(3):202-219.

[33] Van Marrewijk M, Timmers J. Human Capital Management: New possibilities in people management. Journal of Business Ethics 2003;44:171-184.

[34] Beer M. How to develop an organization capable of sustained high performance: Embrace the drive for results-capability development paradox. Organizational Dynamics 2001;29(4):233 - 247.

[35] Seltzer K, Bentley T. The Creative Age: Knowledge and Skills for the new economy. USA: Demos Books, 1999 